\title{
EDITORIAL
}

\section{Flexible excellence}

A short distance from my office there is an enterprise whose slogan is 'flexible excellence'. This signals a determination to deliver quality products in a manner that responds to the variety and changing nature of customers' needs.

Both quality and flexibility are important requirements for survival in today's competitive and turbulent markets. Of course, quality has always been important as a differentiating strategy, and the past twenty years or so have seen an increasing emphasis on managing quality. A flood of management literature on total quality management has educated managers about the role of quality and how it can be built into the firm's products and processes. Additionally there has been a growing recognition that pursuit of quality products and service need not compromise cost efficiency, and moreover, that guaranteeing quality and dependability may even be prerequisites to becoming cost efficient.

Historically, flexibility has received rather less attention as a competitive issue, particularly since flexibility has been seen as in conflict with requirements for efficient, large volume, product-specific production processes. However, flexibility is becoming an increasing concern for firms facing shortening product life cycles and increasing market/demand fluctuations. This requires a response involving increased variety of products and volume fluctuations which must be both timely and cost effective. As with quality and efficiency, managers need to develop an appreciation of the role of flexibility and how it can be built into products and processes. As with quality and efficiency, the potential conflict between flexibility and efficiency needs to be addressed. A recent study has suggested that Japanese manufacturers appear to be working towards cost efficient flexibility in a number of ways: introducing flexible manufacturing systems, reducing lead times in production, developing new processes for new products, reducing set up times and giving workers a broader range of tasks (De Meyer et al, 1989).

Flexibility is a useful attribute for an organisation operating in an uncertain environment. Different aspects of flexibility are apparent in related concepts such as adaptability, resilience, robustness, agility and versatility. Resilience and robustness suggest an emphasis on ability to cope with uncertainty and withstand shocks without fundamentally changing products and processes. Concepts like adaptability, agility and versatility suggest an ability to respond more positively when faced with a changed environment. Described in these terms, flexibility is an attribute which can enable an organisation to react to developments. But flexibility and as sociated concepts can also describe an ability to prepare for and manage an uncertain future in a more proactive way. Thus flexibility can involve actions before or after events and involve either defensive or offensive measures. From this perspective Evans (1991) identifies four archetypal kinds of manoeuvre which provide the means to achieve strategic flexibility:

Pre-emptive manoeuvres - creating options, inflicting surprise or seizing initiatives;

Protective manoeuvres - insuring against losses, hedging, or creating buffers against adverse conditions;

Corrective manoeuvres - the ability to recover from adverse situations and learn from mistakes;

Exploitive manoeuvres - capitalising on opportunities and consolidating advantages.

The key challenge, argues Evans, is 'to develop the capability and deploy available assets to execute swiftly all four types of manoeuvre, individually or collectively, depending on the environmental contingencies and stakeholder preferences in play at a given time'.

In a turbulent, competitive environment the need is for rapid response to changing business conditions. This raises concerns about such things as: introducing new products rapidly and on schedule, improving the quality of sales forecasting and competitor information, keeping up-to-date with relevant technological developments, and successful implementation of new technology. Some observers argue that today's conditions require an emphasis on innovation and a continuous search for and discovery of 
new products and new process solutions. In this environment firms rely on recovering the high costs of innovation through premium pricing of innovatory, differentiated products.

However, an increasing number of firms are not facing this high cost - premium price 'invention' environment, but an environment which Boynton and Victor (1991) call 'dynamic stability'. This environment is characterised by rapid changes in customer demand for products which call for 'revolutionary' product changes but where more 'evolutionary' process technologies are feasible. An important implication is that such firms are better defined in terms of the specific process expertise they bring to the competitive market rather than by the products and services they produce at any given time. The basis for competition is no longer particular products but the knowledge the firm possesses, develops, and exploits.

Firms facing a dynamically stable environment need to develop appropriate flexibility. In particular process capabilities need to become more general purpose, reusable, generic and stable. Processes which are highly efficient only for specific products are inappropriate in this environment. Boynton and Victor argue that these firms need to enhance their information systems to provide not only wider market information, but also information about existing competences and capabilities located throughout the firm. In particular horizontal systems are needed to improve coordination, integration and control of core capabilities and expertise across a variety of functional areas.

Much of the ability of firms to offer high product variety with relatively low process variety is due to the development of flexible techniques in manufacturing. This flexibility can take a number of forms (Chen et al, 1992):

machine based - quick set ups and ability to perform different operations;

materials handling - systems can cope with different parts of different sizes and shapes;

process - given parts may be produced using different processes, materials or operation sequences;

routing - more than one route is possible to produce given parts;

labour - workforce is able to perform a variety of manufacturing tasks effectively and efficiently;

programming - production system is able to operate untended for a certain period of time.
It would seem that the introduction of flexibility in manufacturing processes is a necessary condition for a firm to have the capability to vary products, volumes, and mix of products, or what might be termed 'market flexibility'. For example, computer controlled processes can increase the versatility and speed of operations, permit greater customisation of products and open up new markets. However, manufacturing flexibility is unlikely to be a sufficient condition for market flexibility. Appropriate flexibility in a firm's infrastructure, information systems and market intelligence will also be important.

As a number of writers have noted, the acquisition of flexibility is not without costs, and these need to be compared with the likely benefits. The costs may involve hardware, software, training, reorganisations, and ongoing costs. The benefits in competitive terms are likely to be very hard to quantify, but the necessary strategic assessment must be undertaken. Firms need to determine what form and level of flexibility they should develop. This requires an appreciation of the available options and an effective means of evaluating and implementing them.

Stephen Ward

\section{For the interested reader}

Boynton A C and Victor B (1991): Beyond flexibility: building and managing the dynamically stable organisation, California Management Review, Fall, pp 53-66.

Chen J J, Calantone R J and Chung C-H (1992): The marketingmanufacturing interface and manufacturing flexibility, Omega, 20(4), pp 431-443.

DeMeyer A, Nakane J, Miller J G and Ferdows K (1989): Flexibility: the next competitive battle - the manufacturing futures survey', Strategic Management Journal, 10, pp 135-144.

Evans J S (1991): Strategic flexibility for high technology manoeuvres: a conceptual framework', Journal of Management Studies, 28(1), pp 69-89. 